\title{
Interface Reasoning Research Based on Manifestation Pattern \& Cognitive Load
}

\author{
Long Ren ${ }^{1, \text { a }}$, Zhang Yiting ${ }^{2, b}$ and Wang Yuhui ${ }^{3, c^{*}}$ \\ ${ }^{1}$ Huazhong University of Science and Technology, China \\ ${ }^{1}$ Huazhong University of Science and Technology, China \\ ${ }^{1}$ Huazhong University of Science and Technology, China

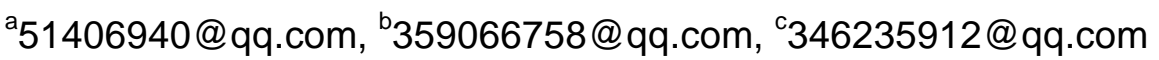

\begin{abstract}
Keywords: Manifestation pattern, cognitive load, graphic reasoning, reasoning mechanism
Abstract. Based on the theory of graphic reasoning, this paper carries out exploration and research on the influence of mental model manifestation pattern and cognitive load theory in interface design reasoning mechanism. It puts forward that users are affected by the mutual influence of mental model manifestation pattern and cognitive load in logical reasoning of graphical interface. It also does task reasoning experiment through the setting of three variables including the logic classification on experimental materials, mental mode manifestation pattern and cognitive load and finally verifies this conjecture. This is of great important significance to reveal the roots of human intelligence and the development of human intelligence potential and it is helpful to improve the availability of interface design.
\end{abstract}

\section{Introduction}

With the rapid development of modern communication technology and internet technology, information communication and information transmission tend to be globalized, highly accelerated and diversified. Although people communicate with each other mostly in verbal or non-schematization form (spoken language, words and numbers, etc) in their daily workplace, in fact, most of functions of human brain are used to process visual information [1]. As important window of information product design and important carrier of information presentation, the visual design of information products plays a bigger and bigger role in social culture dissemination.

GUI(Graphical user interface)is the main carrier of information dissemination. Therefore, when people acquire information, they have higher and higher requirements on the effectiveness, fault tolerance and completion efficiency of interface information acquisition as well as user satisfaction, etc. All of the above can be collectively referred to as interface availability.

The cognition of visual information is the first way for users to do information processing. Cognition is divided into broad-sense cognition and narrow-sense cognition. Broad-sense cognition means that the individuals understand the nature and law of objective things with forms like feeling, presentation, imagination, memory and thinking. It is a kind of cognitive activities. Narrow-sense cognition means that the individuals do processing, storage and extraction of information after acquiring information. The essence of this process is to convert and compile signals of objects into the concept in human brain and it is a creative process. [2]

Reasoning is the core of human intelligence. It is generally divided into abstract reasoning and image reasoning [3]. This paper will not do any research on abstract reasoning. It mainly does exploration and discussion based on the reasoning mechanism of image reasoning. And the theory of image reasoning mainly includes the theory of graphic reasoning.

So in interface design, the study on image reasoning that is on the basis of the manifestation pattern of mental model and cognitive load is of great important significance to reveal the roots of human intelligence and the development of human intelligence potential and is helpful to improve the availability of interface design. 


\section{Related Work}

Manifestation Pattern of Image Reasoning. In the field of cognition, the cognitive psychologist Johnson Laird took advantage of mental model to illustrate how people comprehend language elaboration and daily reasoning. He believes that mental model plays a core and unified role in description of objects and events and it is the basic structure of human cognition. Its cognitive process inputs information for the acceptance of perception and after that it converts them into abstract codes for identification. Mental model utilizes cognition and habits to implement expected inference for sensory information and is an internal representation of a system. The graphical interface is organized through forms such as information architecture, graphics and words. Thus mental models should be also mapped in such a way. Hence manifestation pattern is the external embodiment of mental model.

Most of our cognition on the world around depends on our mental models. Basically all concepts, behavioral process and rules in people's consciousness and facts and experiences in memory can be attributed to mental models. In the field of products, for product design domain, the impact of mental models on cognitive process of products can be divided into three progressive levels: form, function and ultimate goal. Form is composed of elements like shape, materials, structure, color and sound, etc. It is the foundation of the existence of the two levels of function and ultimate goal. [4]

Therefore, the manifestation pattern of mental models can incorporate layout, colors, symbols, graphics, information classification, copywriting, contrast, background and so on. The experimental material design below can be also reflected from the above elements.

Cognitive Load. Cognitive load theory was proposed by John Sweller in the 1980s for the purpose of analyzing the process of learning and solving problems from the perspective of resource allocation. Sweller insists that all kinds of cognitive processing activities in the process of problem solving and learning need to consume cognitive resources. If the total amount of resources required by all activities exceeds the entire amount of resources owned by individuals, the insufficient allocation of resources will be caused and as a result, the efficiency of individual learning and problem solving will be affected. This condition is called cognitive overload [5]. This theory divides cognitive load into the following three types.

1. Intrinsic. The term intrinsic cognitive load was firstly put forward by Chandler and Sweller [6]. It refers to the inherent difficulty level of specific teaching topic. The difficulty of this teaching topic will not change because of teachers.

2. Extraneous. Extraneous cognitive load is generated according to the different ways of information presented to learners. This load is closely bound up with teaching materials. For instance, when there are two possible ways, namely the concept of square can be shown to students through either square graphics or merely semantic description. In this case, the conveyance of visual media is more highly efficient since it will not load too much unnecessary information to students. This kind of unnecessary cognitive load is just extraneous cognitive load.

3. Germane. Germane cognitive load refers to the load that processes, constructs and automatically creates outline. Cognitive load has three characteristics, respectively subjectivity, tacitness and variability. Subjectivity mainly means that different learners have different knowledge base, cognitive styles, processing abilities and personality characteristics, etc. Therefore, there are also differences in task load felt by them and mental efforts put into on them. Consequently the high and low level of cognitive load can reflect larger individual differences; tacitness refers to the phenomenon that can't be seen and touched by learners and cognitive processing process occurs inside the brain; and variability means cognition will change with the change of time and cycle. The structure chart of cognitive load is shown as follows (Table 1): 


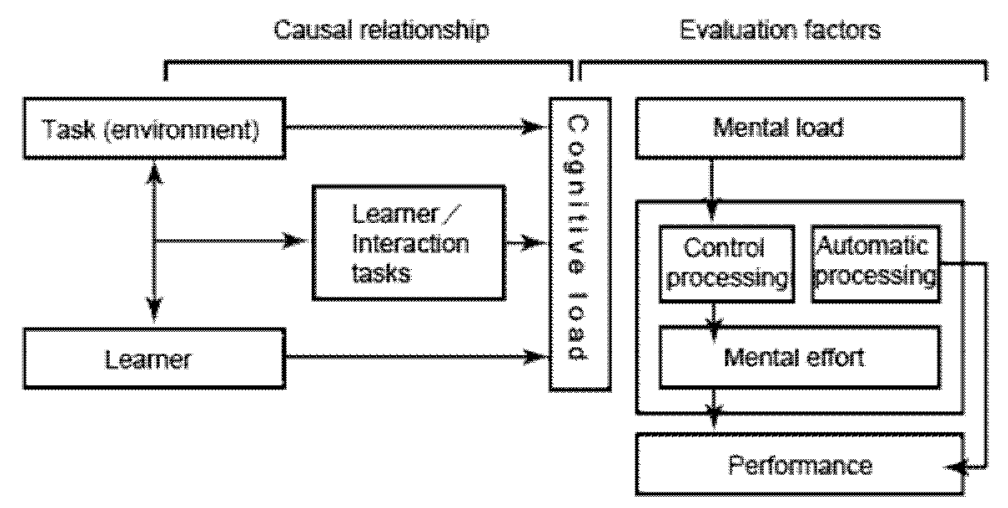

Table 1 The structure chart of cognitive load

Graphic Reasoning. Graphic reasoning is just a thinking process in which one or several known graphics are used to reason some other graphics or information. It can also be regarded as the cognitive activity [8] that reasons and supplements the patterns and symbols of vacant parts in large graphics according to the change rule of patterns or symbols in a pair of large graphics.

Graphic reasoning is a comprehensive thinking process that takes reasoning as dominant factor and other thinking ways as complementary factor; the effectiveness of graphic reasoning not only takes the link of graphics with spatial structure form into account, but also considers the connection between graphics and contents among graphics; and some conclusions of graphic reasoning are certain while others are uncertain. Graphic reasoning just cultivates divergent thinking. In a graphic reasoning, several known graphics become the premise and what is concluded by premise is called conclusion.

In a graphic reasoning, in addition to premise and conclusion, there is also an important part. That is reasoning requirement. Without reasoning requirement, a graphic reasoning can't be completed. Therefore, a graphic reasoning consists of three elements: premise, reasoning requirement and conclusion. [9]. Compared with the common form reasoning, graphic reasoning has the following four characteristics:

(a) One of the important differences between graphic reasoning and common form reasoning is that graphic reasoning is a comprehensive thinking process that takes reasoning as dominant factor and other thinking ways as complementary factor.

(b) The common form reasoning considers the effectiveness of reasoning mainly from the connection with formal structure while the effectiveness of graphic reasoning not only takes the link of graphics with spatial structure form into account, but also considers the connection between graphics and contents among graphics.

(c) The conclusions of common form reasoning are certain. However, some conclusions of graphic reasoning are certain while others are uncertain.

A set of graphics have different regularities when seen from different perspectives, so the conclusion is not uniquely determined. In graphic reasoning, because regularities can be always generalized if given with a number of graphics with limited quantity, the uncertainty of conclusion is accordingly inevitable.

\section{Hypothesis}

(1) The logical reasoning of graphical interface is affected by mental model manifestation pattern.

(2) The size of cognitive load has a significant impact on logical reasoning of graphical interface.

(3) Mental model manifestation pattern and cognitive load interact with each other in logical reasoning of graphical interface. 


\section{Experimental}

Experimental Objectives. Based on the theory of graphic reasoning in image reasoning, this paper carries out exploration and research on the influence of mental model manifestation pattern and cognitive load theory in interface design reasoning mechanism. It validates that whether users are affected by the mutual influence of mental model manifestation pattern and cognitive load in logical reasoning of graphical interface.

In experimental design, it selects three variables, respectively as the classification of information architecture, mental model manifestation pattern and cognitive load. Users do reasoning according to the organization mode of interface and information architecture of graphical interface, so information architecture variables are adopted; in interface design, the impact of mental models on interface cognitive process can be divided into three progressive levels: form, function and ultimate goal. And form consists of elements like layout, structure and color. Thus the variables of mental model manifestation pattern are adopted; and the task difficulty of graphical interface is embodied by cognitive load, so the third variable is cognitive load. Among them, two levels, the same parent-child relationship reasoning \& different parent-child relationship reasoning (logic classification), navigation $\&$ content (manifestation pattern) and high $\&$ low difficulty (cognitive load) are respectively set under each variable.

Participants. Thirty undergraduate students are selected as participants, with balanced gender proportion. The age is $18 \sim 24$. The eyesight and analytical \& thinking ability of all participants are normal.

Experimental Apparatus and Materials. The experimental apparatus is computer, with 17-inch flat-panel display. The participants respond through keyboard and the whole experimental process is controlled by computer programs from the beginning of experiment, making record of participant response and participant information to the end of experiment. The experimental recording is Response Time. According to the classification of navigation and content(Theresa Neil 2012)(Josh Clark2010), tabs and tile structure are the main experimental material design method. The experimental interface is graphical interactive interface with mobile terminal. In order to reduce the semantic interference of images and text, the user interface replaces text and images with the form of symbols. The graphical interface is the common graphical interface and information organization system uses tree structure. The experiment adopts $4 * 2 * 2$ experimental design in participants, which respectively are interface logical order (4 kinds) seen from the table below, the manifestation pattern of graphical interface (clear navigation \& definite content) and cognition load (high-low). Two premises are expressed through three graphical interfaces and conclusion is expressed with one graphical interface. The four graphical interfaces keep the same style in the composition of information content. Totally there are 16 test questions and they will be finished on desktop computers.

\begin{tabular}{|l|c|c|c|c|}
\hline & Premise & Conclusion & Premise & Conclusion \\
\hline Same Parent-child Relationship & A- B- C & D & A-B-D & C \\
\hline Different Parent-child Relationship & $\boldsymbol{a}_{\mathbf{1}}-\boldsymbol{a}_{2}-\boldsymbol{b}_{\mathbf{1}}$ & $\boldsymbol{b}_{2}$ & $\boldsymbol{a}_{\mathbf{1}}-\boldsymbol{a}_{2}-\boldsymbol{c}_{\mathbf{1}}$ & $\boldsymbol{c}_{2}$ \\
\hline
\end{tabular}

Table 2 The Classification of Information Architecture 


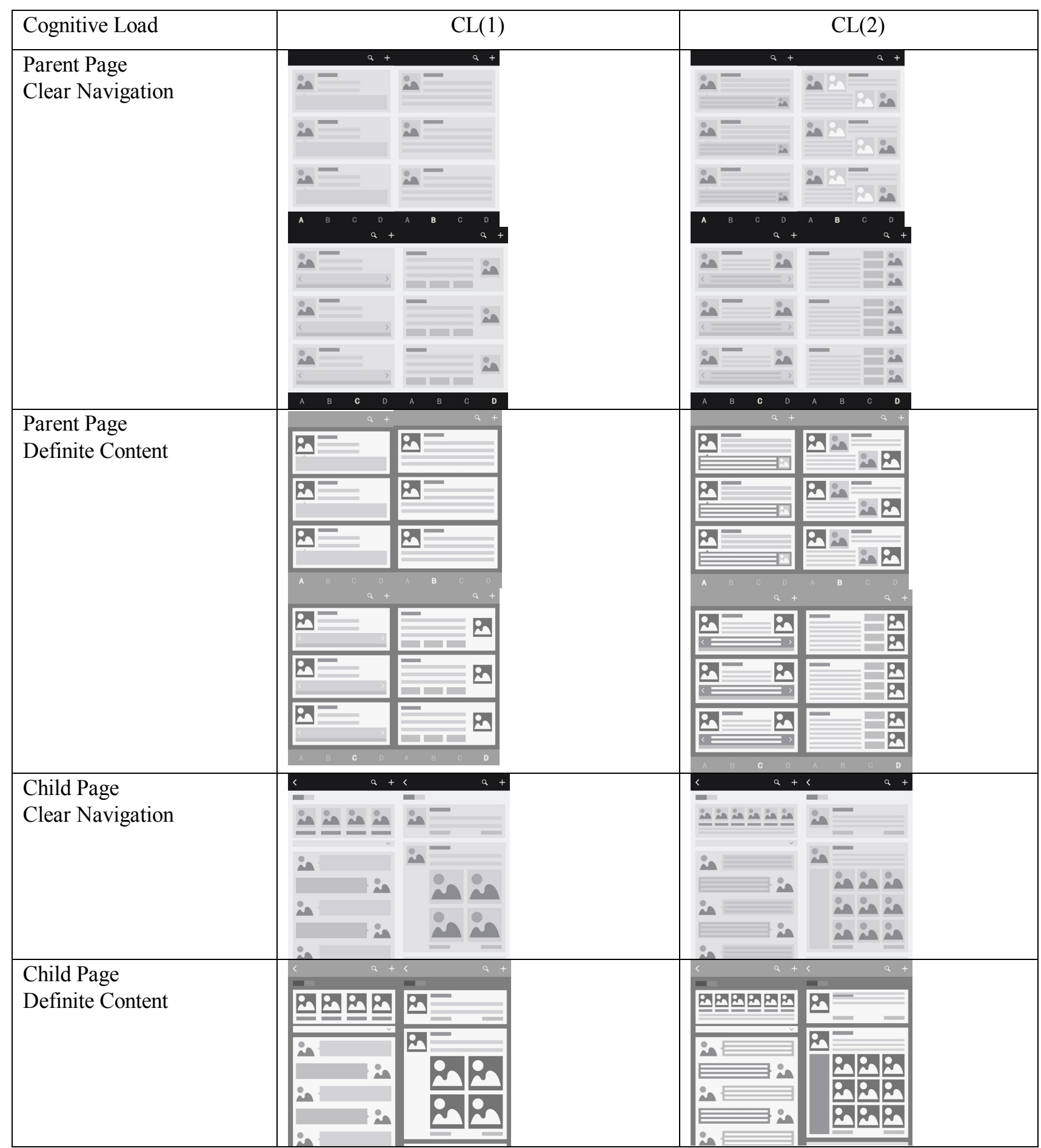

Table 3 The reasoning topics adopted by the experiment

Experimental Procedure. All topics are compiled by E-prime software and they are presented randomly in an experiment. All participants should sit in front of computer and after reading the instructions, they should press the Q key to formally start the test. The experimental steps are as follows: 


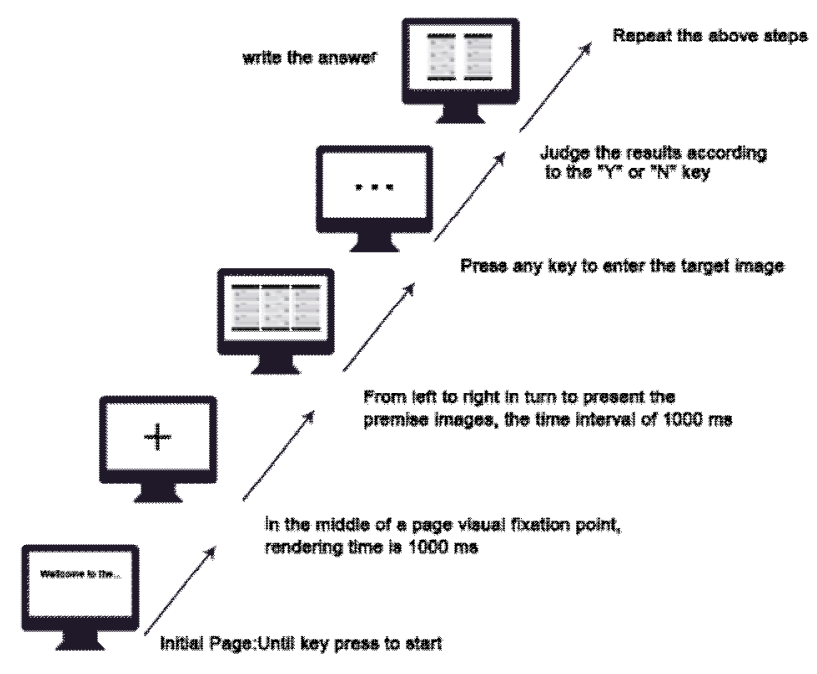

Fig 4 The detailed experimental procedure

The central fixation point will appear in the middle of the screen and three graphical interfaces will appear orderly. After reading them, the participants should press any key to enter the interface of conclusion. The participants are required to judge whether this graphical interface is consistent with the prediction of them or not. If the participants think it is consistent, they shall press Y key. If they think it is unreasonable, they shall press $\mathrm{N}$ key. The participants shall respond very quickly and accurately. The time that makes records from the occurrence of conclusion to key response of participants is the time for conclusion judgment. In order to avoid visual afterimage, the word part of instructions is white and other backgrounds are set black.

All participant traversal experiments use within-subject design. All topics of this experiment are mixed and appear randomly. There are totally 16 test questions. Before the start of formal experiment, firstly participants shall pass three times of exercises and be familiar with experimental mode. The exercises are set randomly according to question types.

Step1. The visual fixation point appears in the middle of a page and the rendering time is $1000 \mathrm{~ms}$.

Step2. The fixation point disappears and three premise pictures are orderly presented from left to right. The time interval is $1000 \mathrm{~ms}$.

Step3. After reading three premise pictures, the participants press any key to enter the target picture.

Step4. The participants do reading and judgment and press " $\mathrm{Y}$ " key or " $\mathrm{N}$ " key according to judgment result.

Step5. Repeat the above steps.

The preliminary screening of experimental results: The raw data is done with preliminary processing to eliminate the data that exceeds mean value by three standard deviations at response time and too disperse participants. In 30 sets of data, the statistical results show that there are a total of three groups of data to be ruled out. The remaining data of 27 participants will be dealt with statistical analysis. 
Results Analysis and discussion.

Dependent Variable: accuracy

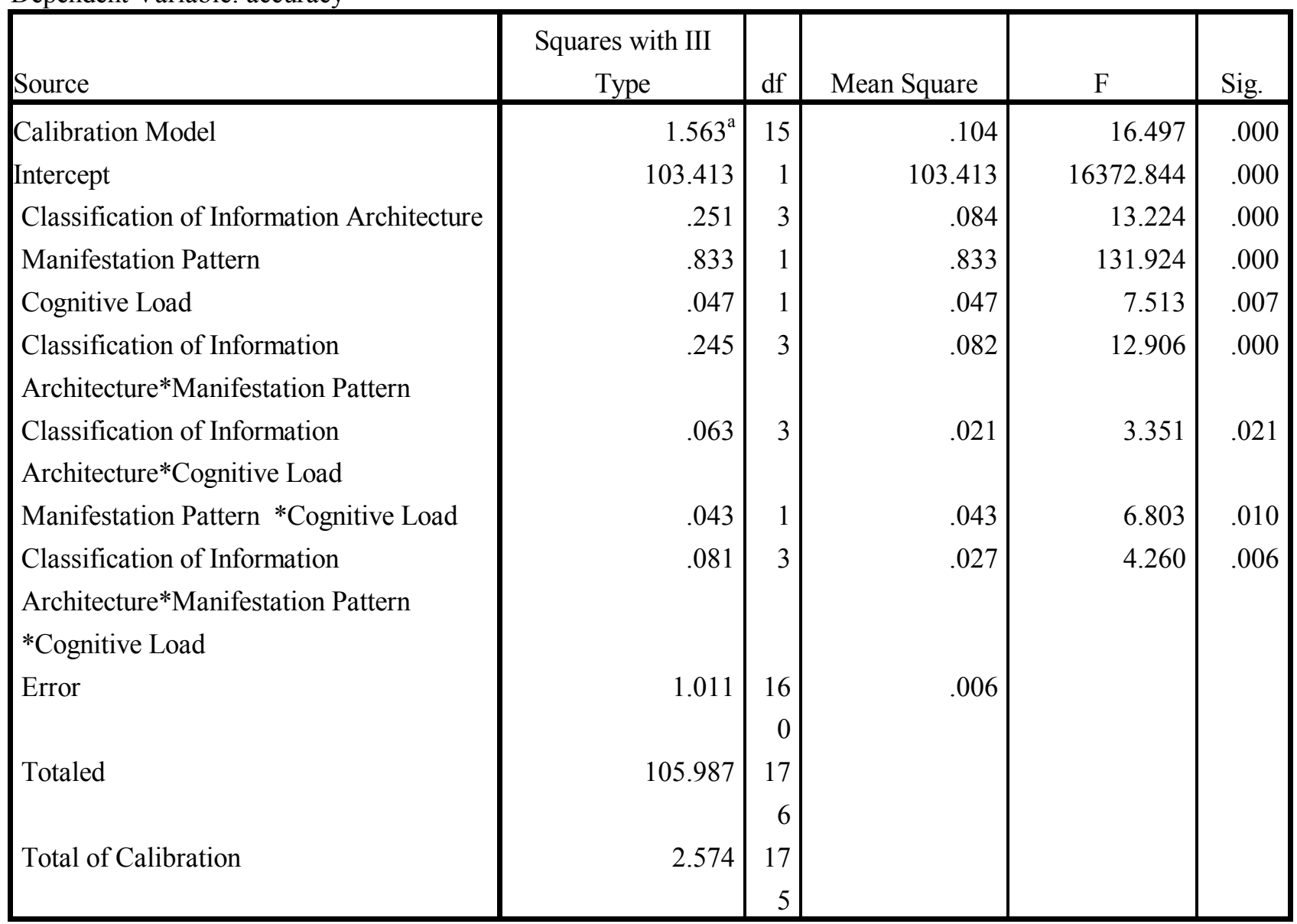

Accuracy

LSD

\begin{tabular}{|c|c|c|c|c|c|c|}
\hline \multirow{2}{*}{$\begin{array}{l}\text { Information } \\
\text { Architecture }\end{array}$} & \multirow{2}{*}{$\begin{array}{l}\text { Information } \\
\text { Architecture }\end{array}$} & \multirow{2}{*}{$\begin{array}{l}\text { Mean } \\
\text { Value-Differe } \\
\text { nt Value (I-J) }\end{array}$} & \multirow{2}{*}{$\begin{array}{c}\text { Standard } \\
\text { Deviations }\end{array}$} & \multirow[b]{2}{*}{ Sig. } & \multicolumn{2}{|c|}{ 95\% Confidence Interval } \\
\hline & & & & & Lower Limit & Upper Limit \\
\hline \multirow[t]{3}{*}{1} & 2 & .0095 & .01694 & .574 & -.0239 & .0430 \\
\hline & 3 & $.0520^{*}$ & .01694 & .003 & .0186 & .0855 \\
\hline & 4 & $.0950^{*}$ & .01694 & .000 & .0615 & .1285 \\
\hline \multirow[t]{3}{*}{2} & 1 & -.0095 & .01694 & .574 & -.0430 & .0239 \\
\hline & 3 & $.0425^{*}$ & .01694 & .013 & .0090 & .0760 \\
\hline & 4 & $.0855^{*}$ & .01694 & .000 & .0520 & .1189 \\
\hline \multirow[t]{3}{*}{3} & 1 & $-.0520^{*}$ & .01694 & .003 & -.0855 & -.0186 \\
\hline & 2 & $-.0425^{*}$ & .01694 & .013 & -.0760 & -.0090 \\
\hline & 4 & $.0430^{*}$ & .01694 & .012 & .0095 & .0764 \\
\hline \multirow[t]{3}{*}{4} & 1 & $-.0950^{*}$ & .01694 & .000 & -.1285 & -.0615 \\
\hline & 2 & $-.0855^{*}$ & .01694 & .000 & -.1189 & -.0520 \\
\hline & 3 & $-.0430^{*}$ & .01694 & .012 & -.0764 & -.0095 \\
\hline
\end{tabular}

Table 5 . Table of LSD Testing 
Among them, the logic classification $\mathrm{F}=13.224, \mathrm{P}=0.000<0.05$. So the main effect of classification of information architecture is significant; manifestation pattern $\mathrm{F}=131.924$, $\mathrm{P}=0.000<0.05$. The main effect of manifestation pattern is significant; cognitive load $\mathrm{F}=7.513$, $\mathrm{P}=0.007<0.05$. The main effect of cognitive load is significant; in the classification of information architecture and manifestation pattern, $\mathrm{F}=12.906, \mathrm{P}=0.000<0.05$. Thus the interaction between classification of information architecture and manifestation pattern is obvious; in the classification of information architecture and cognitive load, $\mathrm{F}=3.351, \mathrm{P}=0.021<0.05$. Therefore the interaction between classification of information architecture and cognitive load is remarkable; in manifestation pattern and cognitive load, $\mathrm{F}=6.803, \mathrm{P}=0.010<0.05$. So the interaction between manifestation and cognitive load is significant; and in the classification of information architecture, manifestation pattern and cognitive load, $\mathrm{F}=4.260, \mathrm{P}=0.006<0.05$. Therefore the interaction among the classification of information architecture, manifestation pattern and cognitive load is significant.

In the table for many comparisons, Level 1, Level 2, Level 3 and Level 4 respectively represents the four logic of variable logical grouping. Level 1 represents the logic A- B- C-D (same parent-child relationship); Level 2 represents the logic A-B-D $-\mathrm{C}$ (same parent-child relationship); Level 3 represents the logic $\mathrm{a}_{\mathbf{1}}-a_{2}-b_{1}-b_{2}$ (different parent-child relationship); and Level 4 represents the logic $a_{1}-a_{2}-c_{1}-c_{2}$ (different parent-child relationship). In Level 1 and Level 2, $\mathrm{P}=0.574>0.05$. So there are no significant differences; in Level 1 and Level 3, P=0.003<0.05. There are obvious differences; in Level 1 and Level 4, $\mathrm{P}=0.000<0.05$. There are significant differences; in Level 2 and Level 3, $\mathrm{P}=0.013<0.05$. There are remarkable differences; in Level 2 and Level 4, $\mathrm{P}=0.000<0.05$. There are significant differences; and in Level 3 and Level 4, $\mathrm{P}=0.012<0.05$. There are obvious differences.

In the above-mentioned experiments, it can be seen that the classification of information architecture, manifestation pattern and cognitive load all have significant effect. This indicates that the accuracy of participants is mainly affected by these three variables.

Among them, the classification of information architecture has interaction with manifestation pattern. Because the external expression of information architecture is restricted by the expression of graphics, words and symbols, the mental model manifestation pattern will produce interference with them when users do identification on information architecture; and there is also interaction between the classification of information architecture and cognitive load. The reason for their obvious interaction is that users carry out the organization, integration and storage of information and so on through working memory when doing identification on information architecture. And this process is influenced by cognitive load. Therefore, cognitive load has played a negative role on users in the process of information classification and information architecture. There is also interaction between mental model manifestation pattern and cognitive load. The visuo-spatial panel will be transformed into working memory when processing graphical information, text information and semantic information. Moreover, mankind only keeps limited information storage capacity and therefore is affected by cognitive load. The increase of manifestation pattern will build up cognitive load, producing cognitive overload.

\section{Summary}

With the growing complexity of information organization mode and information representation in interface design, as advanced processing activity of human, reasoning has produced an important role in the cognitive processing process of graphical interface. The exploration for the impact of manifestation pattern and theory of cognitive load in reasoning mechanism of interface design is very helpful to improve the availability of interface design and is of great important significance to reveal the roots of human intelligence and the development of human intelligence potential.

At the same time, graphical cognition and graphic reasoning will be faced with more and more problems and will cope with more and more difficulties. Graphic reasoning plays the function across time and space. The evaluation system for imperfect graphic reasoning theory, measurement of mental model and manifestation pattern all need to be further studied. 


\section{References}

[1] Robert Horn, Visual Language: Global Communication for the 21st Century, Language Problems \& Language Planning, 1999, 23(3): 299-301(3).

[2] Wu Qinglin. Cognitive Teaching Psychology. Shanghai: Shanghai Scientific and Technical Publishers, 2000.

[3] Du Guoping. Foundation of Classical Logic \& Non-classical Logic. Beijing: Higher Education Press, 2006.

[4] Cheng Dinglv, Study on Symbol Orientation Based on Mental Model in Product Design, Jiangnan University, 2009.

[5] Sweller, J.. Cognitive load during problem solving: Effects on learning. Cognitive science, 1988,12(2), 257-285

[6] Chandler, P., \&Sweller, J. (1991). Cognitive load theory and the format of instruction. Cognition and instruction, 8(4), 293-332.

[7] Sun Chongyong. Measurement of Cognitive Load \& Its Application in Multimedia Learning [J]. PhD thesis of Soochow University, Suzhou, 2012.

[8] Zhang Di \& Zhang Haiyan. Research Status of Graphic Reasoning Ability of Students in Different Stages [J], Journal of Northeast Normal University(philosophy \& social science), 2013(2):232-234

[9] Du Guoping, Graphic Reasoning \& Its Social Culture Function [J], Journal of Jiangsu Normal University (philosophy \& social science). 2011 (1): 123-126

[10] Van der Molen MJ, Henry LA, Van Luit JE.(1Department of Psychology and Education, EMGO Institute for Health and Care Research, VU University, Amsterdam, the Netherlands.), Working memory development in children with mild to borderline intellectual disabilities,Journal of Intellectual Disability Research 2014,58(7):637-650 